\title{
Evaluation on Ethernet based Passive Optical Network Service Enhancement through Splitting of Architecture
}

\author{
C.K. Gomathy, PhD \\ Assistant Professor \\ Department of CSE \\ SCSVMV University-Tamil Nadu.
}

\author{
V. Geetha \\ Assistant Professor \\ Department of CSE \\ SCSVMV University-Tamil Nadu.
}

\begin{abstract}
This Paper Proposes a Survey study of fully distributed Ethernet over Star coupled PON (Passive Optical Network) Architecture. The architecture uses a collision free DBA scheme in which the Optical line Terminal (OLT) is excluded from the implementation of the time slot assignment. To have a distributed architecture, Optical Network Units (ONU's) must be in place without imposing any constraint on the PON Topology. In addition the reliability and performance improvement while using decentralized Ethernet based PON architecture with bandwidth allocation algorithms are discussed.
\end{abstract}

\section{Keywords}

PON Architecture, EPON, Distributed Algorithm, Splitting Architecture.

\section{INTRODUCTION}

Ethernet based Passive optical network (E PON) is certainly a emerging choice for high speed broad band access. A PON is point to Multipoint fiber optical network having no active elements in the signal path, which consist of single, shared optical fiber connecting a service provider's central office box to passive star coupler (SC) which may be located near a residential customer place. The placement of star coupler is purposely made to be far away from central unit but to be near customer residence to minimize and save the fiber. Customers receive a dedicated short optical fiber which connects them to the star coupler but which has a sharing of long distributed trunk fiber. It is obvious that all data transaction is made between Optical line terminal (OLT) and Optical Network Units (ONU's). Any data traffic from OLT to ONU is called down stream (Point to Multipoint), and traffic from ONU to OLT is called Up Stream (Multipoint to point). Two wavelengths are used, Second window wavelength typically $1310 \mathrm{~nm}$ ( $\lambda$ up stream) for the up stream Transmission ( ONU to OLT) and Third window wavelength typically $1550 \mathrm{~nm}(\lambda$ down stream) for down stream transmission ( OLT to ONU).

In the down stream direction, an EPON will operate as a broadcast and perform the operation of network selection. The OLT has the entire bandwidth of the channel to broadcast standard formatted IEEE 802.3 Ethernet frames to all ONU's. Each ONU extracts those packets in transmission link which consist of ONU's Unique MAC (Medium Access Control) address. In the Upstream direction, Multiple ONU's share the transmission channel. Naturally the ONU's has to be given with some arbitration mechanism to avoid collisions. Generally the OLT's arbitrates the upstream transmissions by allocating an appropriate time slot \& Transmission window (TW) to each ONU. An ONU is allowed to transmit any traffic only in time slot of the transmission window by OLT. Within each cycle, in order to inform the OLT about its bandwidth requirements, ONUs use REPORT Messages that are also transmitted along with the data in the TW. Upon receiving a REPORT, the OLT passes the message to a Dynamic Bandwidth Allocation (DBA) module responsible for bandwidth allocation decision. The OLT assigns the TWs via GATE messages.

Several bandwidth allocation schemes have recently been reported in the literature ranging for a static allocation to a dynamically adapting scheme based on instantaneous queue size in every ONU. The simplest is the static TDMA scheme in which every ONU gets a fixed timeslot. While this scheme is very simple; it results in inefficient upstream channel utilization since statistical multiplexing between the ONUs is not possible. A DBA scheme called Interleaved Polling with Adaptive Cycle Time (IPACT) based on Grant and Request messages. This scheme uses an interleaved polling approach where the next ONU is polled before the transmission from the previous one has arrived. This scheme provides statistical multiplexing for ONUs and results in efficient upstream channel utilization.

To date, the mainstream of these EPON bandwidth allocation schemes as well as the new IEEE 802.3ah EFM Task Force specifications have been centralized-relying on a component in the central office (OLT) to provision upstream traffic. Hence, the OLT is the only device that can arbitrate timedivision access to the shared channel. Since the OLT has global knowledge about the state of the entire network, this is a centralized control plane in which the OLT has a centralized intelligence. One of the major problems associated with a centralized architecture is the "single-point of failure problem"; that is the failure of the OLT will bring down the whole access network. It is the purpose of this work to propose a distributed solution to this problem and in the process to prove that, in addition to the added flexibility and reliability, the performance of the proposed decentralized EPON architecture and the associated bandwidth allocation algorithms are at least as efficient as their centralized counter parts.

Specifically, this work proposes a novel Ethernet over Star Coupler-based PON architecture. The architecture uses a fully distributed collision-free DBA scheme in which the OLT is excluded from the implementation of the time slot assignment. To implement a distributed control plane, direct connectivity (communicability) between the ONU's should be in place without imposing any constraint on the PON topology. In the proposed architecture, the ONUs exchange signaling and control information concerning their queue status and their transmission needs amongst themselves.

Then, the ONUs simultaneously and independently run instances of the same DBA algorithm outputting identical bandwidth allocation results. Once the algorithm is run, the ONUs sequentially and orderly transmit their data without any 
collisions, eliminating the OLT's centralized task of processing requests and generating.

A:

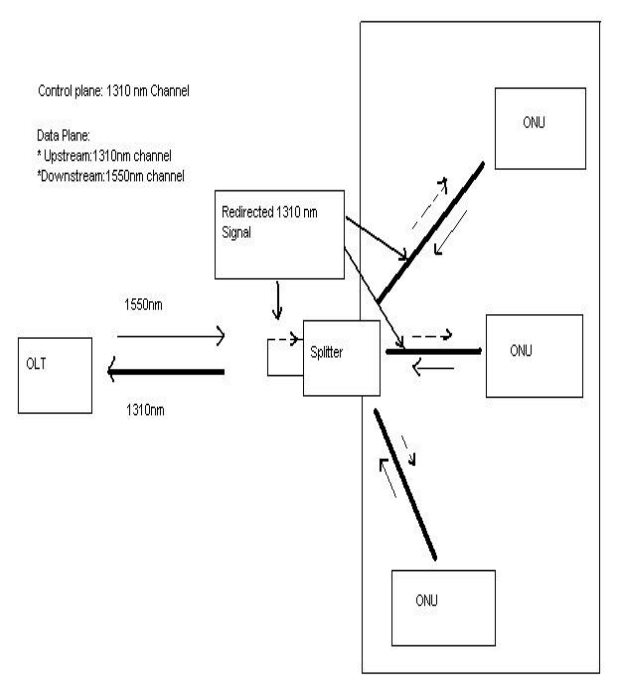

B:

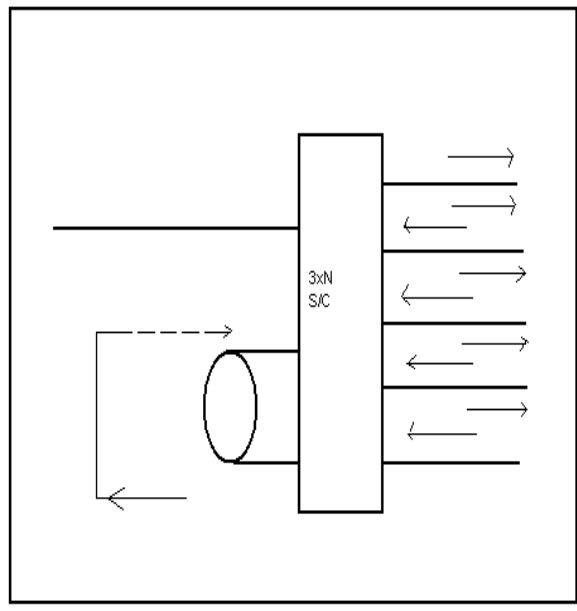

$3 \times N$ SIC

C:

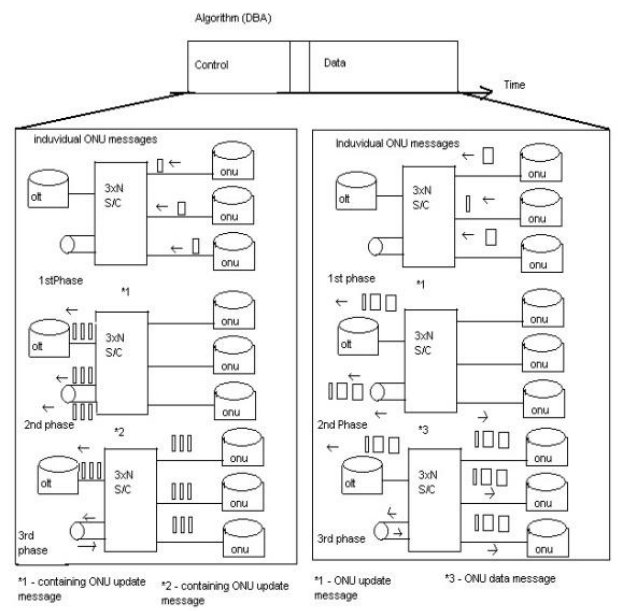

Fig1: A,B,C- Grants for bandwidth assignment

\section{THE PROPOSED SOLUTION - DISTRIBUTED ALGORITHM}

Fig. 1 shows the general architecture of this approach. As can be seen from Fig. 1, a portion of the optical signal power transmitted by an upstream transmitter ( $\lambda$ upstream) toward the OLT will be redirected back and broadcasted to all ONUs. This can be achieved by connecting two ports of a $3 \times \mathrm{N} \mathrm{SC}$ with each other through an optical isolator as shown in Fig. 1. Note that in addition to the conventional transceiver maintained at each ONU (a $\lambda$ up transmitter and a $\lambda d$ receiver), this approach requires an extra receiver tuned at $\lambda$ up. A base band direct detection circuit is needed to detect the redirected control channel ( $\lambda$ up) in order to recover the control update information. This architecture assumes a cycle-based upstream link; a cycle is defined as the time that elapses between two executions of the scheduling algorithm. The cycle size can either have fixed, or variable length (confined within a certain upper bound) to accommodate the dynamic upstream traffic conditions. The cycle is divided into three periods; a static update period (control plane), a fixed waiting period (processing control messages and running the algorithm) and a dynamic transmission period (data plane). The proposed cycle, along with the details of how the control plane performs the updating process is shown in Fig. 2a in three phases. Each ONU transmits its update control message in its own assigned fixed time update slot (first phase). These messages are then combined at the SC and a multiplexed update message is created (second phase). In the third phase, a fraction of the multiplexed control

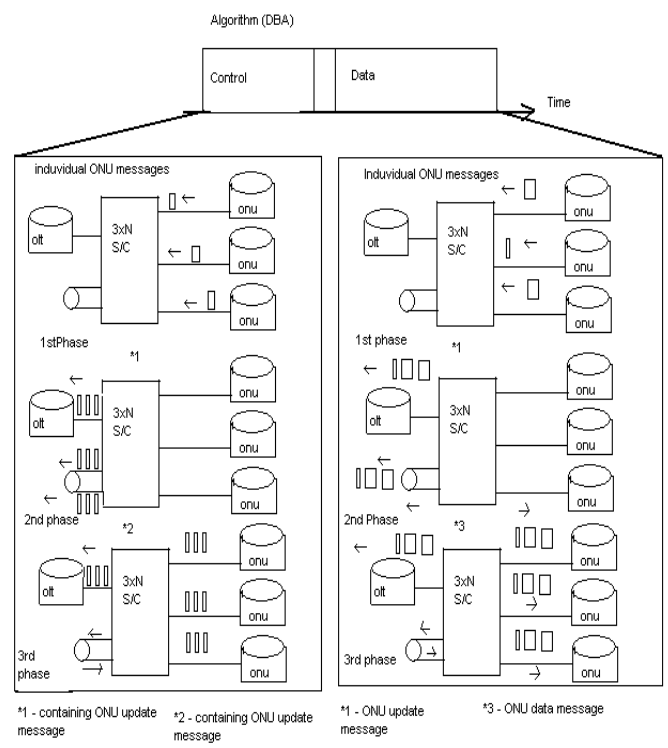

Fig 2: Proposed Distributed EPON a) Cycle updating process b) Transmission process

Signal is transmitted through the first output port of the SC and propagates to the OLT (which could discard it, make use of it as a synchronization message, and/or process the control information).

Another fraction of the multiplexed control signal is redirected back and broadcasted to all ONUs (through the isolator). A base band direct detection circuit located at each ONU is then used to detect the redirected control channel ( $\lambda$ update). The detected signal is then processed in order to Recover the control data information belonging to each of the other (N-1) ONUs. Since there are only two operating communication wavelengths ( $\lambda$ up and $\lambda d$ ), signaling and 
upstream transmission take place on the same communication channel ( $\lambda$ up) and the periods will appear sequentially as on the top of fig 2 .

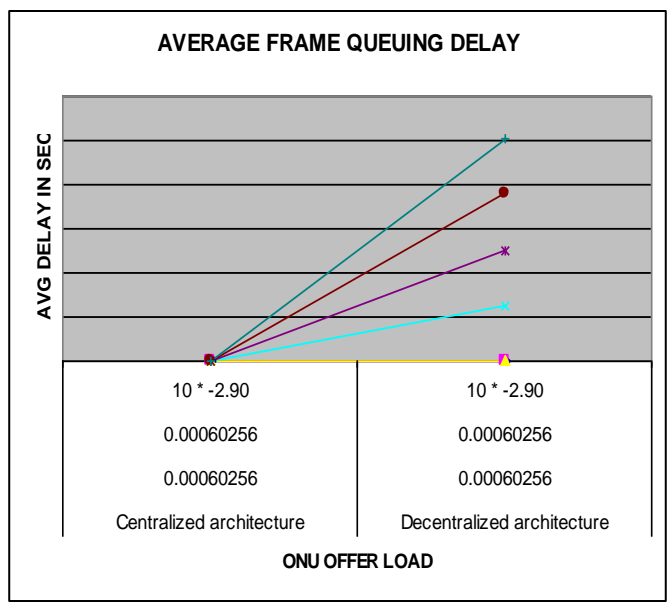

Fig 3: Average Frame Queuing Delay for Centralized and Decentralized architectures.

\subsection{The First Period (Control Plane)}

The update period is divided into $\mathrm{N}$ equal fixed time slots where $\mathrm{N}$ is the number of the ONU stations in the network. The update period is used for the ONUs to communicate their status and to exchange signaling and control message information with one another. Each ONU uses its own fixed time slot within the update period to transmit its control message. For simplicity, and to avoid collisions, the assignment of these $\mathrm{N}$ timeslots follows a fixed TDMA assignment since control messages are fixed in size. Note that the control slots in the proposed distributed scheme are all transmitted sequentially in one period (update period). This in contrast to the centralized schemes where the control slot (REPORT Message) of each ONU is transmitted along with the data in the TW allocated to it by the OLT. All control update messages are transmitted as Ethernet frames. Because the signaling information is segregated from the upstream traffic, signaling information can be timelier and complete thus increasing the efficiency of the Dynamic Bandwidth Allocation algorithm.

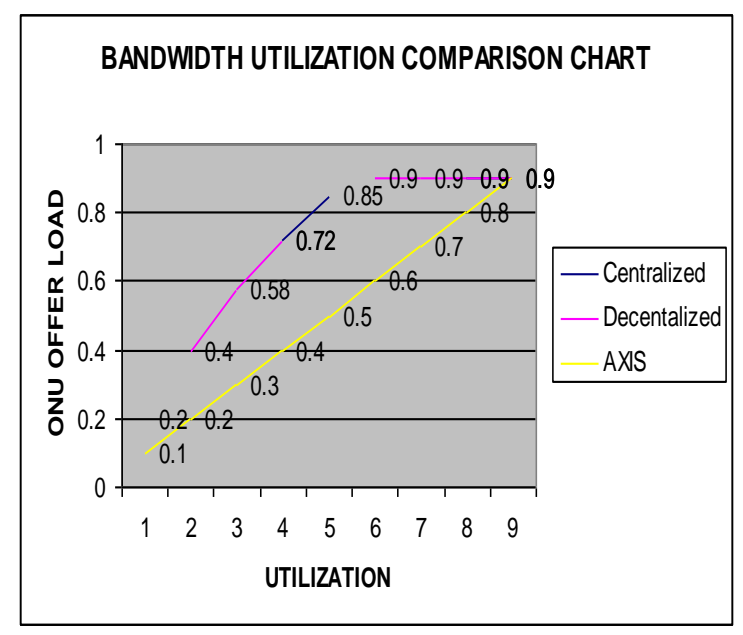

Fig 4: Bandwidth Utilization for Centralized and decentralized architecture

\subsection{The Second Period (Algorithm Execution)}

The second period of fixed length is a waiting period (no upstream transmissions are allowed during this period) and is used for allowing the ONU's to process the information gathered from the multiplexed control message. Each ONU maintains a table with information about the state of the queues at each other ONU. This information is updated each cycle whenever the ONU receives a new multiplexed control message from all other ONUs. The DBA algorithm module uses the table maintained at each ONU. Note that instances of the same DBA algorithm are executed simultaneously and independently at each ONU. An execution of the algorithm yields a unique set of ONU assignments (wi) identically produced in each ONU (wi is the amount of bytes that an ONU is allowed to transmit in its TW). In other words, the algorithm should not incorporate any assumptions or randomness to handle exceptions. This is because several instances of it will run locally and independently at each ONU.

\subsection{The Third Period (Data Plane)}

The third period or (transmission period) is essentially a giant slot used for actual upstream data transmission. During the transmission period, the ONU's follow exactly the allocation scheme the algorithm produced (i.e. their transmissions start at specific times and last for specific bytes) as shown in Fig. $2 b$. Note that the order of ONU's transmission may be different in each cycle and need not be fixed; but rather is a function of the ONU's traffic demand. This is a major advantage compared to the fixed transmission order.

\section{PERFORMANCE EVALUATION}

The traffic model used here is the same as that where each ONU is modeled to be fed by a number of ON/OFF sources, each with a Pareto distribution governing the lengths of the ON/OFF periods, to capture the self-similar nature of Ethernet traffic. To compare the performance results of the proposed decentralized model with that of the centralized scheme (IPACT). we use the same system parameters used therein; a system with 16 ONUs, access link data rate from users to an ONU of $100 \mathrm{Mb} / \mathrm{s}$, and a $1 \mathrm{~Gb} / \mathrm{s}$ upstream link data rate (from an ONU to the OLT). Several bandwidth allocation algorithms were studied. Namely: fixed, limited, gated, constant credit and linear credit. Amongst these algorithms, the limited (where the OLT grants the requested number of bytes, but no more than a given predetermined maximum), was shown to exhibits the best performance. Due to the space limitations, we use the simple limited DBA algorithm for comparing our distributed architecture versus that of the centralized scheme reported therein. Fig. 3 presents the mean frame queuing delay, for both the centralized and distributed architectures using the Limited DBA algorithm, as a function of an ONU's offered load. In the case of the proposed decentralized approach, the order of the transmitting ONUs in a given cycle is not fixed (as in IPACT), but rather ordered based on the allocated TW determined by the DBA algorithm (the highest allocation transmits first; ties are broken by the ONU ID). From the results, it is observed that the decentralized approach improves IPACT in terms of the average frame delay at low loads. This is because by interchanging the order of transmissions, a given ONU's update message is closer in time to its corresponding transmission. Thus, a more current depiction of its buffer status is governing the transmission. As the load increases more ONU's request more than the maximum allowed 
window, and thus more get the same allocation (maximum window). This, in turn, makes the advantage of the interchanged order of transmission to vanish. Fig. 4 shows the channel bandwidth utilization for both the centralized and distributed architectures using the Limited DBA algorithm, as a function of an ONU's offered load. As can be seen from the figure, the performances of the two architectures are almost identical, with the centralized approach exhibiting a slight advantage (less than 1\%). Finally, it is important to emphasize that; in general, distributed architecture-based DBA algorithms (future work) would outperform those of the centralized architecture-based DBA algorithms. This is because a distributed DBA algorithm takes into account all other ONU requests when allocating a TW to a given ONU. This is in contrast to the centralized architecture. Here all the proposed DBA algorithms take into account only that particular individual ONU request when allocating to it.

\section{CONCLUSION}

This work has proposed a novel decentralized Ethernet over Star Coupler-based PON architecture. The performance of the proposed distributed EPON architecture and the associated bandwidth allocation algorithms are shown to be as efficient as their centralized counterpart. While the proposed distributed architecture increases the complexity of the ONU, however, the added flexibility and reliability of such architecture might justify the extra cost. Furthermore, because the signaling information is segregated from the upstream traffic, signaling information can be timelier and complete thus increasing the efficiency of the DBA algorithm. These enhanced DBA algorithms would have the ability to support better QoS Characteristics because transmission of the signaling information is not constrained by the shared data/control upstream channel associated with the centralized schemes.

\section{REFERENCES}

[1] B. Lung, "PON Architecture 'Future proofs' FTTH," Lightwave, vol. 16, no. 10, Sept. 1999, pp. 104-7.

[2] D. Mynbaev, "From Core to Metro to Access Networks The Need for Passive Optical Networks,".

[3] C.K.Gomathy , Dr.S.Rajalakshmi. "A Business Intelligence Network Design for Service-Oriented Architectures", International Journal of Engineering Trends and Technology(IJETT), V9(3),151-154 March 2014. ISSN:2231-5381.

[4] G. Kramer and G. Pesavento, "Ethernet Passive Optical Network (EPON): Building a Next Generation Optical Access Network," IEEE Com. Mag., pp. 66-73, Feb. 2002.

[5] Alloptic, "Ethernet Passive Optical Networks," The International engineering Consortium, http://www.iec.org .

[6] C.K.Gomathy and Dr.S.Rajalakshmi, "Business Process Development In Service Oriented Architecture", International Journal of Research in Computer Application and Management (IJRCM) ,Volume 1,Issue IV, August 2011,P.No:50-53,ISSN : 2231-1009 .

[7] G. Kramer, et al. "Ethernet PON (ePON): Design and Analysis of an Optical Access Network," Photonic Network Communications Journal, vol. 3, No. 3, July 2001.

[8] G. Kramer, B. Mukherjee, and G. Pesavento, "IPACT: A dynamic Protocol for an Ethernet PON (EPON)," IEEE Comm. Mag., pp. 74-80, Feb. 2002. 